\title{
Replication of Papilio maackii Ménétriés butterfly scale structural color using a magnetron sputtering method
}

\author{
WU LiYan ${ }^{1}$, HAN ZhiWu ${ }^{2 *}$, SONG YuQiu ${ }^{1}$, NIU ShiChao ${ }^{2} \&$ Ren LuQuan ${ }^{2}$ \\ ${ }^{1}$ Department of Engineering, Shenyang Agricultural University, Shenyang 110161, China; \\ ${ }^{2}$ Key Laboratory of Bionic Engineering of Ministry of Education, Jilin University, Changchun 130025, China
}

Received April 23, 2012; accepted September 2, 2012

\begin{abstract}
The natural butterfly Papilio maackii Ménétriés scales were found, through scanning and transmission electron microscopy, to comprise stacks of thin layers. Replicated scales were then fabricated on a silicon substrate using a magnetron sputtering (MS) method with ITO $\left(\mathrm{In}_{2} \mathrm{O}_{3}(90 \%)+\mathrm{SnO}_{2}(10 \%)\right)$ and polymethyl methacrylate (PMMA). Finally, the optical effects of the replicated scale were examined by a spectrometer. The results showed that the replicated and natural scales have the similar characteristics in structural colors. As a result, it can be concluded that the MS method reported in this paper is a feasible method for fabricating an artificial biological structural color or other optical elements.
\end{abstract}

butterfly, scale, structural color, magnetron sputtering

Citation: Wu L Y, Han Z W, Song Y Q, et al. Replication of Papilio maackii Ménétriés butterfly scale structural color using a magnetron sputtering method. Chin Sci Bull, 2012, 57: 4525-4528, doi: 10.1007/s11434-011-4898-9

Biological structural colors have been attracting significant attention. Many artifices are employed by creatures to display metallic-like colors as signals, defending against predators, and camouflage [1-3]. Mäthger et al. [4] studied the Paradise whiptail (Pentapodus paradiseus) and found that it has distinct reflective stripes on its head and body. These reflective stripes contain a dense layer of physiologically active iridophores, which act as multilayer reflectors. McPhedran et al. discussed two examples of living creatures using photonic crystals to achieve structural colors: the sea mouse (Aphroditidae Polychaeta), which has a hexagonal close packed structure of holes in its spines; and the jelly fish (Bolinopsis infundibulum), which has an oblique array of high index inclusions in its antennae [5]. Other studies found that many animals have structural color effects such as Urania moth, African starling feathers [6], Cosmophasis spider [7], and Bostrychia hagedash bird [8].

Butterflies are experts in producing the structural color phenomena by various means [9-13], including reflection, diffraction, and interference, etc. [14-17]. Vukusic et al. [18]

*Corresponding author (email: zwhan@jlu.edu.cn) reported that the wings of the African swallowtail butterfly can absorb the UV component of incident light by a pigment, and the wing scales with finely spaced micro-holes can prevent the loss of iridescence. Tada et al. [19] observed the butterfly $P$. blumei and found different structural colors at different viewing angles. Their results showed that colors changing from green to purple due to non-planar specula reflection. Parker reviewed animals' structural colors and divided them into different types based on the optical mechanism [20-22]. Recently, some studies have demonstrated that a photonic crystal structure in butterflies can also produce structural color [23,24].

The multiple reflection system of butterfly scales display shininess and variable colors. Researchers in the bionics field have expended considerable effort attempting to replicate these natural structures in order to develop new optical devices [25-28]. However, the mass production of manmade structural color scales is highly complex and difficult to be obtained.

In this study, the microstructure of scales of butterfly Papilio maackii Ménétriés was observed by scanning electron microscopy (SEM) and transmission electron micros- 
copy (TEM). Based on the microstructural characteristics, a replicated scale was fabricated using a magnetron sputtering (MS) method. The optical characteristics of the natural scale and the replicated scale were examined by spectrometer. The structural color effect of the replicated scale showed good similarity to the natural scale.

\section{Materials and methods}

\subsection{SEM experiment}

The specimens for SEM experiments were taken from the blue parts of the Papilio maackii Ménétriés dorsal wings. The specimens were then rinsed with ether to eliminate grime, grease and proteins on the wing surfaces, and treated by a series of dehydration operations. Before observation, the dehydrated specimens were pasted onto a metallic testbed and cold sputtered with gold.

\subsection{TEM experimental specimen}

The specimen was taken from the same parts of the Papilio maackii Ménétriés dorsal wings. The specimen was treated by the following major steps: (i) it was immersed in $4 \%$ glutaraldehyde for $2 \mathrm{~h}$ to avoid structural changes due to water evaporation from the dried cells; (ii) it was placed in sodium cacodylate buffer for $1.5 \mathrm{~h}$; (iii) it was kept in $1 \%$ osmic acid for $1.5 \mathrm{~h}$ and dehydrated through an ethanol series; (iv) it was embedded in epoxy resin and solidified in an oven for $4 \mathrm{~h}$; (v) it was sliced into lamellas with a thickness of $70 \mathrm{~nm}$.

\subsection{Magnetic sputtering}

The replicated scale was fabricated using JGP-450A (Shenyang Scientific Instruments Develop Center, CAS) multitarget magnetron sputtering equipment. The targets materials were $\mathrm{In}_{2} \mathrm{O}_{3}(90 \%)+\mathrm{SnO}_{2}(10 \%)$ (ITO) and polymethyl methacrylate (PMMA) with refractive indices of 2.0 and 1.5 respectively. The substrate was silicon slice. First, the substrate was ultrasonically cleaned in acetone, ethanol, and deionized water solution for $10 \mathrm{~min}$ respectively. The slice was then placed under vacuum for drying at a temperature of $250^{\circ} \mathrm{C}$ for $1 \mathrm{~h}$. During the sputtering process, the flow rate of $\mathrm{Ar}$ was maintained at $60.0 \mathrm{sccm}$. The electric current for target ITO was $0.5 \mathrm{~A}$ and the power for PMMA was 100 $\mathrm{W}$. By imitating the natural scales microstructure, the two materials were alternately sputtered on the substrate. The quality and thickness of sputtering layer is directly related to the sputtering speed and time. In order to obtain a favorable sputtering layer, experimental determination of the appropriate sputtering speeds was adopted. The sputtering time was temporarily set as $1 \mathrm{~h}$ for both ITO and PMMA. Figure 1 shows the method for the calculation of sputtering speeds for two materials, in which the sputtered specimens

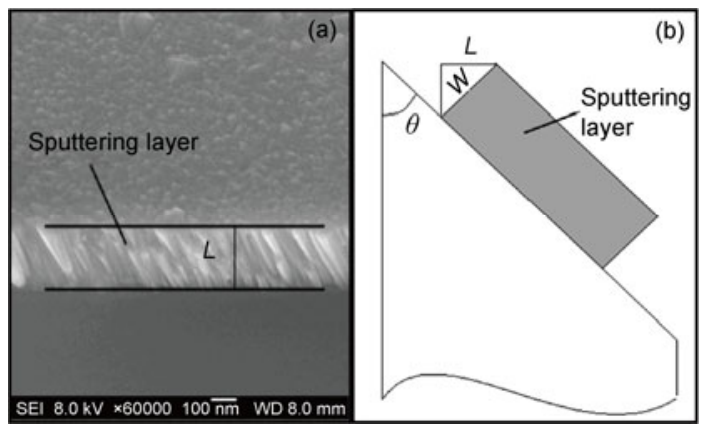

Figure 1 Measurement of sputtering speeds. (a) Sputtering layer under SEM; (b) calculation scheme for sputtering speeds.

were pasted on metal beds at an angle of $\theta=45^{\circ}$ for SEM observation. The dimensions measured under SEM, $L$, were measured, and the measurements were repeated 3 times for each material and the mean values were recorded. The sputtering speeds were then calculated through eq. (1). Finally, the sputtering speeds were determined to be 0.106 $\mathrm{nm} / \mathrm{s}$ for ITO and $0.15 \mathrm{~nm} / \mathrm{s}$ for PMMA.

$$
v=\frac{W}{t}=\frac{L}{t \cos \theta},
$$

where, $W$ is the layer thickness (nm), $t$ is sputtering time (s), and $\theta$ is the observation angle $\left(^{\circ}\right)$.

With the sputtering speeds and expected thickness of sputtering layer, the sputtering time for each target can be readily obtained, and the whole process was controlled by computer.

\subsection{Spectrometer measurement}

The reflectivities of the natural and replicated scales were analyzed using a TU-1901 spectrometer over wavelengths ranging from 350 to $800 \mathrm{~nm}$. The light sources were tungsten and deuterium lamps for normal incidence.

\section{Results and discussion}

Figure 2(a) shows the region where the specimens were taken. Figure 2(b) is the SEM image of the overlapping arrangement of scales on the upper surface of the wing. Figure 2(c) presents the high-magnification TEM image of a single scale microstructure in cross-section. It can be seen that the scale is characterized by a series of evenly spaced parallel lamellar features, which are responsible for the multiple reflection and interference events that cause the structural color effect. The reflector can be simplified to a schematic model, as illustrated in Figure 2(d).

Base on the results from SEM and TEM, replication of Papilio maackii Ménétriés butterfly scales was accomplished by the MS method. The thickness of single layer of ITO and PMMA was set at $120 \mathrm{~nm}$ that means the sputtering time for 


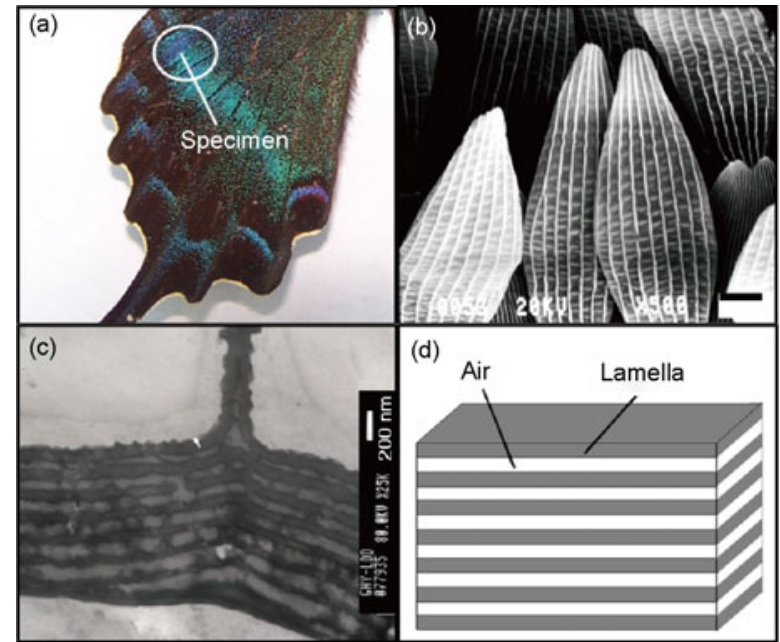

Figure 2 Structural color scales of Papilio maackii Ménétriés and the stacks model. (a) The shining blue scales were cut down for SEM and TEM experiments; (b) SEM image of the natural scales; (c) the crosssection image under TEM; (d) schematic of a one-dimensional model.

ITO was $1132 \mathrm{~s}$ and that for PMMA was $800 \mathrm{~s}$.

The two targets material lamellae were alternately sputtered onto the substrate. One periodicity consisted of one ITO and one PMMA layer. Figure 3 shows the processed replicated scale with 5 periodicities, which appears a shining blue color. The detailed optical effect of the replicated scale was examined by a spectrometer.

Figure 4 presents the spectrometer experimental results. The first peak reflectivity wavelength of the natural scale appears in the range 440-460 $\mathrm{nm}$ (Figure 4(a)) and the maximum is 0.7 . Figure 4(b) shows that the reflectivity peak wavelength of the replicated scale appears at about $450 \mathrm{~nm}$ and the maximum is only 0.4 . The location of peak means the replicated scale and natural ones have the same structural color effect, i.e. blue. However, the maximum reflectivity of the replicated structure was lower than the natural ones. This difference may have been due to the absorbance effect of both the sputtering and substrate materials or the accuracy factor in the dimensional measurements of the SEM and TEM images. In addition to the difference in the maximum reflectivity, the natural scale reflectivity shows a second peak at about $800 \mathrm{~nm}$ which is not present in the replicated scales. However, this wavelength falls into

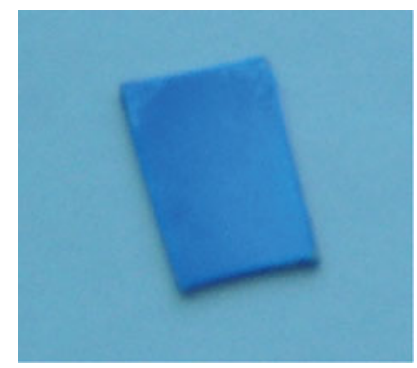

Figure 3 Finished replicated scale fabricated by the MS method.
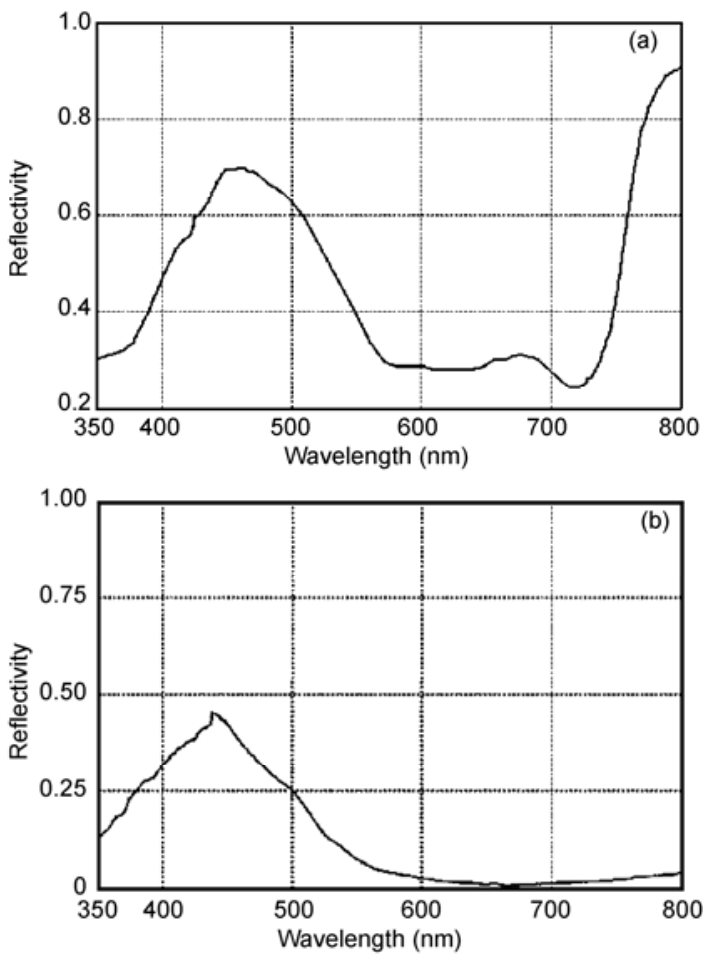

Figure 4 Structural color effect contrast of the replicated and natural scales. (a) Reflectivity curve of the original Papilio maackii Ménétriés wing scales; (b) reflectivity curve of the replicated scale.

the infrared spectrum, which cannot be perceived by naked eyes. In other words, both replicated and natural scales shows a blue structural color that we can actually see.

\section{Conclusion}

The microstructural characteristics of the natural scales of butterfly Papilio maackii Ménétriés were observed and analyzed by SEM and TEM. Based on the observation results, the microstructure was simplified to a one dimensional multilayer structure, and this structure was successfully fabricated through MS method. The optical effect of the replicated scale was examined with spectrometer. Results show that the color features of the replicated scale were in reasonable agreement with those of the natural scales, which shows that the MS technique is a feasible method for replicating the structural color effect of the Papilio maackii Ménétriés wing. Also, an inference could be made that the MS method has considerable potential for the fabrication of other natural structural colors and high-performance optical elements.

This work was supported by the National Natural Science Foundation of China (50635030), the Specialized Research Fund for the Doctoral Program of Higher Education (20100061110023), Ministry of Education, China, the Scientific and Technological Development Project of Jinlin University (20111808) and the Open Project of the Key Lab of Bionic Engineering (Jilin University) (K201003). 
1 Tan T L, Wong D, Lee P. Iridescence of a shell of mollusk Haliotis Glabra. Opt Express, 2004, 12: 4847-4854

2 Li B, Zhou J, Li L T, et al. One-Dimension photonic crystal structure in the abalone carapace. Chinese Sci Bull, 2005, 13: 1422-1424

3 Parker A R, Mckenzie D R, Large M C J. Multilayer reflectors in animals using green and gold beetles as contrasting examples. J Exp Biol, 1998, 201: 1307-1313

4 Mäthger L M, Land M F, Siebeck U E, et al. Rapid colour changes in multilayer reflecting stripes in the paradise whiptail, pentapodus paradiseus. J Exp Biol, 2003, 206: 3607-3613

5 McPhedran R C, Nicorovici N A, McKenzie D R, et al. Structural colours through photonic crystals. Physica B, 2003, 338: 182-185

6 Parker A R. A vision for natural photonics. Phil Trans R Soc Lond A, 2004, 362: 2709-2720

7 Parker A R, Hegedus Z. Diffractive optics in spiders. J Opt A: Pure Appl Opt, 2003, 5: S111-S116

8 Brink D J. VanderBerg N G. Structural colors from the feathers of the bird Bostrychia hagedash. J Phys D: Appl Phys, 2004, 37: 813-818

9 Wong T H, Gupta M C. Color generation in butterfly wings and fabrication of such structures. Opt Lett, 2003, 28: 2342-2344

10 Vukusic P, Sambles J R. Color effects in bright butterflies. JSDC, 2000, 116: 376-380

11 Brink D J, Lee M E. Confined blue iridescence by a diffracting microstructure: An optical investigation of the Cynandra opis butterfly. Appl Opt, 1999, 38: 5282-5289

12 Brink D J, Smit J E, Lee M E, et al. Optical diffraction by the microstructure of the wing of a moth. Appl Opt, 1995, 34: 6049-6057

13 Plattner L. Optical properties of the scales of Morpho rhetenor butterflies: Theoretical and experimental investigation of the backscattering of light in the visible spectrum. J R Soc Interface, 2004, 1: 49-59

14 Liao G. L, Zuo H B, Cao Y B, et al. Optical properties of the micro/ nano structures of Morpho butterfly wing scales. Sci China Tech Sci, 2010, 53: 175-181

15 BiróL P, Bálint Z, Kertész K, et al. Role of photonic-crystal-type structures in the thermal regulation of a Lycaenid butterfly sister species pair. Phys Rev E, 2003, 67: 0219071

16 Prum R O, Quinn T, Torres R H. Anatomically diverse butterfly scales all produce structural colours by coherent scattering. J Exp Biol, 2006, 209: 748-765

17 Kinoshita S, Yoshioka S, Kawagoe K. Mechanisms of structural color in the Morpho butterfly: Cooperation of regularity and irregularity in an iridescent scale. Proc R Soc Lond B, 2002, 269: 1417-1421

18 Vukusic P, Sambles J R, Lawrence C R. Color mixing in wing scales of a butterfly. Nature, 2000, 404: 457

19 Tada H, Mann S E, Miaoulis I N, Wong P Y. Effects of a butterfly scale microstructure on the iridescent color observed at different angles. Opt Express, 1999, 5: 87-92

20 Parker A R. 515 million years of structural color. J Opt A: Pure Appl Opt, 2000, 2: 15-28

21 Parker A R, Martini N. Structural color in animals-simple to complex optics. Opt Laser Technol, 2006, 38: 1-8

22 Kertész K, Bálint Z, Vértesy Z, et al. Photonic crystal type structures of biological origin: Structural and spectral characterization. Curr Appl Phys, 2006, 6: 252-258

23 Li B, Li Q, Zhou J, et al. Photonic structures in butterfly Thaumantis diores. Chin Sci Bull, 2004, 49: 2545-2546

24 Ren L Q, Qiu Z M, Han Z W, et al. Structural color in butterfly Apatura Ilia scales and the microstructure simulation of photonic crystal. J Bionic Eng, 2008, 5(Suppl): 14-19

25 Qin Y H, Liu F, Yin H W, et al. Photonic structure in the wings of Papilio Banor Gnesa. Chin Sci Bull, 2007, 52: 3183-3188

26 Kang S H, Tai T Y, Fang T H. Replication of butterfly wing microstructures using molding lithography. Curr Appl Phys, 2010, 10: 625-630

27 Wang L, Sun H F, Zhou H H, et al. Preparation of BST nanocrystals embedded in $\mathrm{SiO}_{2}$ film by magnetron sputtering for nonvolatile memory applications. Chin Sci Bull, 2011, 56: 1139-1141

28 Tang N, Wang J L, Xu H X, et al. Optical characterization of ZnO thin films deposited by RF magnetron sputtering method. Sci China Ser E-Tech Sci, 2009, 52: 2200-2203

Open Access This article is distributed under the terms of the Creative Commons Attribution License which permits any use, distribution, and reproduction in any medium, provided the original author(s) and source are credited. 